\title{
Association of COVID19-affected Mental Health with Menstrual Abnormalities Among University Students: A Cross sectional study from Pakistan.
}

\author{
Ayaz Ali Samo ${ }^{1 *}$, Nimra Masood Baig ${ }^{1}$, Raheela Bibi Sayed ${ }^{2}$, Aisha Sohail ${ }^{1}$, Fazila Usman ${ }^{1}$, Sayeda Jeea \\ Abbas ${ }^{1}$, Tayyaba Khan ${ }^{1}$, Zulfiqar Ali Laghari ${ }^{1}$.
}

1. Department of Physiology and Medical Laboratory Technology University of Sindh, Jamshoro, Sindh, Pakistan.

2. Govt. Nazareth Girls Degree College Hyderabad, Sindh Pakistan.

Received: December 02, 2021 Accepted: December 12, 2021 DOI: $10.46568 /$ bios.v2i2.55

\begin{abstract}
:
Introduction: Mental health is crucial for wellbeing. COVID-19 pandemic has caused mental health problems. In this study it was hypothesized that COVID19 related mental health disturbances may have impacted the menstrual health. Objective of this study was to evaluate the impact of COVID-19 affected mental health on the menstrual health of university students. Methods: A cross sectional study was conducted at University of Sindh, Jamshoro between July to August 2021 during fourth wave. A pretested questionnaire was administered to collect the data. Healthy unmarried female students were included in the study. Mental health parameters were assessed using DAS Scale. Results: For a total 400 study participants the mean age was $20.82 \pm 1.69$. The prevalence of mild to severe depression, mild to severe anxiety, and mild to moderate stress was $46.3 \%, 62 \%$ and $29.3 \%$ respectively. In moderately depressed females there was an increased prevalence of oligomenorrhea $\left[\chi^{2}=34.5 \mathrm{p}<0.0001\right]$, heavy menstrual blood flow $\left[\chi^{2}=21.8 \mathrm{p}<0.001\right]$, increased duration of menstrual bleeding $\left[\chi^{2}=21.56\right.$ $\mathrm{p}<0.001]$, and heavy menstrual dysmenorrhea $\left[\chi^{2}=18.78 \mathrm{p}<0.002\right]$. In moderately anxious females there was an increased prevalence of oligomenorrhea $\left[\chi^{2}=15.5 \mathrm{p}<0.07\right]$, heavy menstrual blood flow $\left[\chi^{2}=12.4\right.$ $\mathrm{p}<0.03]$, and increased duration of menstrual bleeding $\left[\chi^{2}=21.56 \mathrm{p}<0.001\right]$. In moderately stressed females there was increased prevalence of polymenorrhagia $\left[\chi^{2}=22.3 \mathrm{p}<0.001\right]$, increased duration of menstrual bleeding $\left[\chi^{2}=24.23 \mathrm{p}<0.0001\right]$, heavy menstrual dysmenorrhea $\left[\chi^{2}=21.28 \mathrm{p}<0.01\right]$ and secondary amenorrhea $\left[\chi^{2}=4.28 \mathrm{p}<0.01\right]$. Conclusion: COVID19-affected Mental Health affected menstrual health. Healthcare system of Pakistan should address this issue while planning to mitigate the impacts of COVID-19 on human health.
\end{abstract}

Keywords: COVID-19, Mental Health, Menstrual abnormalities, Students, Pakistan

*Correspondence: Ayaz Ali Samo: Department of Physiology and Medical Laboratory Technology, University of Sindh, Jamshoro, Pakistan. Phone: +92 229213227 Fax: +92 22 9213227, Email: ayazsamo@ usindh.edu.pk

\section{Introduction}

Coronavirus infectious disease 2019 (COVID-19) is caused by Severe Acute Respiratory Syndrome Coronavirus 2 (Sars-CoV2). COVID-19 started in Wuhan, China in December 2019. The rapid rise in COVID19-related morbidities and mortalities led to declaration of global public health emergency by World Health Organization (WHO)[1]. Since November 2019, the COVID19 pandemic has affected not only people who needed healthcare but also the normal population. With an increase in COVID-19 cases, number of preventive measures were taken by nations to contain the COVID-19 infections, these measures include imposition of nationwide lockdowns, establishment of COVID-19 isolation centers, staying at home to avoid infection and social distancing[2]. Closure of offices, shopping malls and public parks, unavailability of 
transportation, and entertainment centers have disturbed the psychosocial health of people[3]. Several studies have reported COVID19 related increased in depression, anxiety, stress, changes in sleep patterns, generalized anxiety disorder, bereavement, isolation, making females more vulnerable to such pandemic related problems[4-8]

Psychological factors may affect reproductive health of females[9] Previously conducted studies have shown an association between mental health problems and female menstrual cycle. Hormonal balance plays a crucial role in maintaining moods and emotions, fertility, pregnancy and menstrual cycle[10, 11]. Increasing evidence suggests that females with premenstrual syndrome (PMS), premenstrual dysphoric disorder (PMDD), and irregular periods have increased mental health problems such as anxiety, severe depression, and stress[12, 13]. A previously conducted study has shown women with symptoms of depression and a perceived stress, these women have an increased prevalence of irregular menstrual cycles[14]. A recently conducted online survey based study from 60 countries indicates that younger adults are at greater risk of mental health problems due to COVID19 pandemic[15].Debowska et al, have shown that female university students are at more risk of depression, anxiety, and stress[16]. This study also reported that compared with students (aged $\geq 25$ ) the students (aged 18-24) were at more risk of mental health problems[16]. Study from Greece also indicated that females were at more risk of developing COVID19-related stress, anxiety, and depression[17]. Previously published prepandemic studies on Menstrual Abnormalities from Pakistan [18,19] give an insight on prevalence of Menstrual abnormalities. Dars et al, using a sample of 401 study participants have reported on Menstrual Abnormalities, they have shown that $76 \%$ of females were normal, $13 \%$ had oligomenorrhea, 7\% had polymenorrhagia, 4\% had irregular menses[18]. Ali et al, using a sample of 1285 adolescent girls have reported that the blood flow of $67 \%$ of females was normal, $6.5 \%$ experienced heavy blood flow. Growing body of evidence indicates that COVID-19 has impacted mental health and has a relationship with increased prevalence of Menstrual Abnormalities. Post pandemic studies from Turkey and Jordan indicated that females reported having undefined Menstrual abnormalities during COVID-19 pandemic[20]. A multinational study has shown that $25 \%$ of females have increased cycle length, $20 \%$ have decreased cycle length, 36\% reported that they noticed changes in bleeding pattern[21], Another report indicated a positive association of COVID-19 pandemic and increased frequency of missing cycles[22].

However, to the best of our knowledge there is no single study published from Pakistan to show the impact of COVID-19 affected mental health on menstrual abnormalities. We tried to assess the impact of COVID19 affected mental health on menstrual abnormalities.

\section{METHODS:}

\section{Study design:}

This cross-sectional study was carried out during months of July and August 2021 at University of Sindh, Jamshoro. This university is the second oldest educational institution of Pakistan where more than 25000 students are pursuing their education in various disciplines.

\section{Sample size:}

Population size of the University of Sindh, Jamshoro is 25000 students, by hypothesizing 50\%+/5 frequency of outcome factor in the population of 25000 students at $95 \%$ confidence interval, 379 samples were enough to carry out study. The sample size was calculated using EPI software version $3[23]$.

\section{Data collection:}

Data was collected through online mode. Questionnaire was distributed using social media platforms including WhatsApp, Facebook, Telegram, Twitter, and E-mails. Questionnaire designed for collecting data consisted of 3 sections including demographic information, mental 
health information and menstrual cycle related information. Questionnaire was translated in Urdu language for clarity of study participants. We used the Urdu version of depression, anxiety, and stress scale (DASS-21) downloaded from http://www2.psy.unsw.edu.au/dass/Urdu/Aslam.htm [24]. Cronbach alpha test value for DASS Scale was 0.84. The first page of the online questionnaire was about participant's informed consent to participants in the study in which they were assured about secrecy and confidentiality of personal data.

\section{Inclusion and Exclusion criteria:}

Data of healthy unmarried female students at University of Sindh, Jamshoro aged (17-25 year), without any known gynecological disorder were included in the final analysis. Females who were married, students from other universities, age $>25$, with any known gynecological, mental or any other disorder were excluded from analysis.

\section{Variable setting:}

The demographic information included age and year of study of study participants. DASS-21 scale is a self-report questionnaire used to screen depression, anxiety, and stress. DASS-21 consists of twenty-one items, seven items for each subscale. Participants answered the question by encircling the number from 0 to 3 which indicates severity of a condition from never to almost always. Questions including did COVID-19 pandemic affect your mental health? Did COVID19 pandemic affect your menstrual cycle? Have you ever experienced early or late periods before your date in stressful conditions? Did menstrual problems affect your academic performance? were set as categorical with answers yes or no. Menstrual Abnormalities were defined as: secondary amenorrhea: no period during the last 3 months. Menstrual bleeding flow was determined by the number of pads used by study participants. Menstrual bleeding time was determined by the number of days the bleeding lasted.

\section{Statistical analysis:}

The excel sheet of data collected online was retrieved. Then, a master excel sheet were generated, that was transferred to Special Package for Social Sciences Software (SPSS) version 23. Data was edited as per inclusion criteria. Descriptive statistics was used to calculate the frequencies. Chi square test was used to associate between 2 factors and outcomes. Cronbach alpha test was used to assess the reliability score of scale data. $\mathrm{P}$ value $<0.05$ were considered for as significant.

\section{RESULTS:}

\section{Basic characteristics of study participants:}

A total of 400 students participated in this study. The minimum age was 17 years and maximum age was 25 years. The mean age of study participants was $20.82 \pm 1.69$. Among 400 study participants $82(20.5 \%)$ were from Undergraduate class I, $96(24 \%)$ were from Undergraduate class II, 83 (20.75\%) were from Undergraduate class III, 139 (34.81\%) were from Undergraduate class IV. Among all the study participant 59.75\% responded that COVID-19 has affected their mental health, $25.75 \%$ participants replied that their menstrual health has been affected due to COVID-19, $65 \%$ of respondent replied that they have experienced early or late periods, $11.25 \%$ had oligomenorrhea $15.25 \%$ had polymenorrhagia $10.75 \%$ had no fixed patterns of menstrual cycle. When a question about flow of bleeding was asked $16.75 \%$ participants experienced light bleeding flow, $68.25 \%$ participants experienced moderate bleeding menstrual bleeding flow $15 \%$ of participants experienced heavy blood flow. When were participants asked how long their bleeding lasted? Three percent of participants replied that their bleeding days lasted for $\leq 2$ days, $89.5 \%$ participants replied that their bleeding lasted for 3-7 days, $24.2 \%$ participants replied that their bleeding days lasted for 7days, and $7.5 \%$ participants replied that their bleeding days lasted for $>7$ days. When participants were asked about severity after menstrual dysmenorrhea, $29.25 \%$ 
participants said that they experience mild dysmenorrhea, $40.25 \%$ participants said that they experienced moderate dysmenorrhea, and $21.75 \%$ participants said that they experienced severe dysmenorrhea during menses. Sixteen percent participants had secondary amenorrhea. Thirty six percent of study participants said that their academic performance have been affected to the menstrual problems. Among the 400 participants prevalence of mild to severe depression $46.6 \%$. The prevalence of mild to extremely severe anxiety was $62.1 \%$. The prevalence of mild to moderate stress was $29.3 \%$ (Table 1).

Table 1. Association of mental health problems with menstrual abnormalities.

\begin{tabular}{|c|c|}
\hline+2 & Frequency \\
\hline $\begin{array}{l}\text { Age Group in years } \\
18-20 \\
21-25\end{array}$ & $\begin{array}{l}181(45.3 \%) \\
219(54.8 \%)\end{array}$ \\
\hline $\begin{array}{l}\text { Class } \\
\text { Undergraduate I } \\
\text { Undergraduate II } \\
\text { Undergraduate III } \\
\text { Undergraduate IV }\end{array}$ & $\begin{array}{l}82(20.5 \%) \\
96(24 \%) \\
83(20.75 \%) \\
139(34.81 \%)\end{array}$ \\
\hline $\begin{array}{l}\text { Did COVID-19 pandemic affect your mental health? } \\
\text { No } \\
\text { Yes }\end{array}$ & $\begin{array}{l}161(40.25 \%) \\
239(59.75 \%)\end{array}$ \\
\hline $\begin{array}{l}\text { Did COVID19 pandemic affect your menstrual cycle? } \\
\text { No } \\
\text { Yes }\end{array}$ & $\begin{array}{l}297(74.25 \%) \\
103(25.75 \%)\end{array}$ \\
\hline $\begin{array}{l}\text { Have you ever experienced early or late periods before your date in } \\
\text { stressful condition? } \\
\text { No } \\
\text { Yes }\end{array}$ & $\begin{array}{l}140(35 \%) \\
260(65 \%)\end{array}$ \\
\hline $\begin{array}{l}\text { Menstrual Disorder } \\
\text { Normal } \\
\text { Oligomenorrhea } \\
\text { Polymenorrhagia } \\
\text { No fixed Pattern } \\
\end{array}$ & $\begin{array}{l}251(62.75 \%) \\
45(11.25 \%) \\
61(15.25 \%) \\
43(10.75 \%)\end{array}$ \\
\hline $\begin{array}{l}\text { Number of pads used } \\
1 \\
2-5 \\
>5\end{array}$ & $\begin{array}{l}67(16.75 \%) \\
273(68.25 \%) \\
60(15 \%)\end{array}$ \\
\hline $\begin{array}{l}\text { Days of menstrual bleeding usually during each period? } \\
\leq 2 \\
3-7 \\
>7\end{array}$ & $\begin{array}{l}12(3 \%) \\
358(89.5 \%) \\
30(7.5 \%)\end{array}$ \\
\hline $\begin{array}{l}\text { Dysmenorrhea } \\
\text { No dysmenorrhea } \\
\text { Mild dysmenorrhea } \\
\text { Moderate dysmenorrhea } \\
\text { Heavy dysmenorrhea } \\
\end{array}$ & $\begin{array}{l}35(8.75 \%) \\
117(29.25 \%) \\
161(4025 \%) \\
87(21.75 \%)\end{array}$ \\
\hline $\begin{array}{l}\text { Secondary Amenorrhea } \\
\text { No } \\
\text { Yes }\end{array}$ & $\begin{array}{l}334(83.5 \%) \\
66(16.5 \%)\end{array}$ \\
\hline $\begin{array}{l}\text { Did menstrual problems affect your academic performance? } \\
\text { No } \\
\text { Yes }\end{array}$ & $\begin{array}{l}144(36 \%) \\
256(64 \%)\end{array}$ \\
\hline Mental Health Prob & \\
\hline
\end{tabular}


Biosight 2021; 02(02): 40-49

Mild to Severe Depression

Mild to Extreme Anxiety

$185(46.6 \%)$

$248(62.1 \%)$

Mild to Moderate Stress

$117(29.3 \%)$

Association of categories of depression with Menstrual Abnormalities:

Among study participants who responded that COVID19 pandemic has impacted their menstrual cycle there was an increased prevalence of moderate depression $\left[\Sigma^{2}=8.74 \mathrm{p}\right.$ value $\left.<0.03\right]$. $\left.<0.001\right]$. In moderately depressed females there was an increased prevalence of oligomenorrhea $\left[\Sigma^{2}=34.5\right.$ $\mathrm{p}<0.0001]$, heavy menstrual blood flow $\left[\Sigma^{2}=21.8 \mathrm{p}<0.001\right]$, increased duration of menstrual bleeding $\left[\Sigma^{2}=21.56 \mathrm{p}<0.001\right]$, and severe dysmenorrhea $\left[\Sigma^{2}=18.78 \mathrm{p}<0.002\right]$. The academic performance of moderately depressed females was also disturbed $\left[\Sigma^{2}=4.02 \mathrm{p}\right.$ value $\left.<0.05\right]$ (Table 2).

Table 2. Association of categories of depression with Menstrual Abnormalities.

\begin{tabular}{|c|c|c|c|c|c|c|}
\hline Factors & Frequency & $\begin{array}{l}\text { Normal } \\
\mathrm{n}=215 \\
(53.8 \%)\end{array}$ & $\begin{array}{l}\text { Mild } \\
\mathrm{N}=90 \\
(22.5 \%)\end{array}$ & $\begin{array}{l}\text { Moderate } \\
\mathrm{n}=82 \\
(20.5 \%) \\
\end{array}$ & $\begin{array}{l}\text { Severe } \\
\mathrm{n}=13 \\
(3.3 \%) \\
\end{array}$ & $\begin{array}{l}\Sigma^{2} \text { and } \mathrm{p} \\
\text { value }\end{array}$ \\
\hline $\begin{array}{l}\text { Did COVID-19 pandemic } \\
\text { affect your mental health? } \\
\text { No } \\
\text { Yes }\end{array}$ & $\begin{array}{l}161 \\
(100 \%) \\
239 \\
(100 \%)\end{array}$ & $\begin{array}{l}95(59 \%) \\
120(50 \%)\end{array}$ & $\begin{array}{l}30(18.6 \%) \\
60(25.1 \%)\end{array}$ & $\begin{array}{l}28 \\
(17.4 \%) \\
54 \\
(22.6 \%)\end{array}$ & $\begin{array}{l}8(5 \%) \\
5(2.1 \%)\end{array}$ & 0.07 \\
\hline $\begin{array}{l}\text { Did COVID19 pandemic } \\
\text { affect your menstrual } \\
\text { cycle? } \\
\text { No } \\
\text { Yes }\end{array}$ & $\begin{array}{l}297 \\
(100 \%) \\
103 \\
(100 \%) \\
\end{array}$ & $\begin{array}{l}170 \\
(52.2 \%) \\
45(43.7 \%)\end{array}$ & $\begin{array}{l}67(22.6 \%) \\
23(22.3 \%)\end{array}$ & $\begin{array}{l}52(17.5 \%) \\
30 \\
(29.1 \%)\end{array}$ & $\begin{array}{l}8(2.7 \%) \\
5(4.9 \%)\end{array}$ & $\begin{array}{l}8.74 \\
\mathbf{0 . 0 3}\end{array}$ \\
\hline $\begin{array}{l}\text { Have you ever experienced } \\
\text { early or late periods before } \\
\text { your date in stressful } \\
\text { condition? } \\
\text { No } \\
\text { Yes }\end{array}$ & $\begin{array}{l}140 \\
(100 \%) \\
260 \\
(100 \%)\end{array}$ & $\begin{array}{l}92(65.7 \%) \\
123(47.3 \%)\end{array}$ & $\begin{array}{l}28(20 \%) \\
62(23.8 \%)\end{array}$ & $\begin{array}{l}18(12.9 \%) \\
64(24.6 \%)\end{array}$ & $\begin{array}{l}2(1.4 \%) \\
11(4.2 \%)\end{array}$ & $\begin{array}{l}14.67 \\
\mathbf{0 . 0 0 2}\end{array}$ \\
\hline $\begin{array}{l}\text { Menstrual Disorder } \\
\text { Normal } \\
\text { Oligomenorrhea } \\
\text { Polymenorrhagia } \\
\text { No fixed Pattern }\end{array}$ & $\begin{array}{l}251(100 \%) \\
45(100 \%) \\
61(100 \%) \\
43(100 \%)\end{array}$ & $\begin{array}{l}150(59.8 \%) \\
15(33.3 \%) \\
25(41 \%) \\
25(58.1 \%)\end{array}$ & $\begin{array}{l}53(21.1 \%) \\
8(17.8 \%) \\
16(26.2 \%) \\
13(30.21 \%)\end{array}$ & $\begin{array}{l}44(17.5 \%) \\
20(44.4 \%) \\
15(24.6 \%) \\
3(7 \%)\end{array}$ & $\begin{array}{l}4(1.6 \%) \\
2(4.4 \%) \\
5(8.2 \%) \\
2(4.7 \%)\end{array}$ & $\begin{array}{l}34.52 \\
<0.0001\end{array}$ \\
\hline $\begin{array}{l}\text { Menstrual bleeding flow } \\
1 \\
2-5 \\
>5\end{array}$ & $\begin{array}{l}67(100 \%) \\
273 \\
(100 \%) \\
60(100 \%)\end{array}$ & $\begin{array}{l}25(37.3 \%) \\
163(59.7 \%) \\
27(45 \%)\end{array}$ & $\begin{array}{l}23(34.3 \%) \\
57(20.9 \%) \\
10(16.7 \%)\end{array}$ & $\begin{array}{l}15(22.4 \%) \\
48(17.6 \%) \\
19(31.7 \%)\end{array}$ & $\begin{array}{l}4(6 \%) \\
5(1.8 \%) \\
4(6.7 \%)\end{array}$ & $\begin{array}{l}21.68 \\
\mathbf{0 . 0 0 1}\end{array}$ \\
\hline
\end{tabular}

Association of categories of anxiety with Menstrual Abnormalities:

Among study participants who responded that COVID19 pandemic has impacted their mental health there was an increased prevalence of moderate and severe anxiety $\left[\Sigma^{2}=19.09 \mathrm{p}\right.$ value 
<0.001]. Among study participants who responded that COVID19 pandemic has impacted their menstrual cycle there was an increased prevalence of moderate and severe anxiety $\left[\Sigma^{2}=15 \mathrm{p}\right.$ value $<0.005]$. In moderately anxious females there was an increased prevalence of oligomenorrhea $\left[\Sigma^{2}=15.5 \mathrm{p}<0.07\right]$, heavy menstrual blood flow $\left[\Sigma^{2}=12.4 \mathrm{p}<0.03\right]$, and increased duration of menstrual bleeding $\left[\Sigma^{2}=21.56 \mathrm{p}<0.001\right]$. The academic performance of moderately depressed anxious females was also disturbed $\left[\Sigma^{2}=14.37 \mathrm{p}\right.$ value $\left.<0.006\right]$ (Table 3).

Table 3. Association of categories of Anxiety with Menstrual Abnormalities

\begin{tabular}{|c|c|c|c|c|c|c|}
\hline Factors & Frequency & $\begin{array}{l}\text { Normal } \\
\mathrm{n}=152 \\
(38 \%)\end{array}$ & $\begin{array}{l}\text { Mild } \\
n=49 \\
(12.3 \%)\end{array}$ & $\begin{array}{l}\text { Moderate } \\
\mathrm{n}=131 \\
(32.8 \%)\end{array}$ & $\begin{array}{l}\text { Severe } \\
\mathrm{n}=68 \\
(17 \%) \\
\end{array}$ & $\begin{array}{l}\Sigma^{2} \\
\text { and } p \\
\text { value }\end{array}$ \\
\hline $\begin{array}{l}\text { Did COVID-19 pandemic } \\
\text { affect your mental health? } \\
\text { No } \\
\text { Yes }\end{array}$ & $\begin{array}{l}161(100 \%) \\
239(100 \%)\end{array}$ & $\begin{array}{l}76(47.2 \%) \\
76(31.8 \%)\end{array}$ & $\begin{array}{l}17(10.6 \%) \\
32(13.4 \%)\end{array}$ & $\begin{array}{l}43(26.27 \%) \\
88(36.8 \%)\end{array}$ & $\begin{array}{l}25(15.5 \%) \\
43(18 \%)\end{array}$ & $\begin{array}{l}9.98 \\
\mathbf{0 . 0 1}\end{array}$ \\
\hline $\begin{array}{l}\text { Did COVID19 pandemic } \\
\text { affect your menstrual } \\
\text { cycle? } \\
\text { No } \\
\text { Yes }\end{array}$ & $\begin{array}{l}297(100 \%) \\
103(100 \%)\end{array}$ & $\begin{array}{l}125(42.1 \%) \\
27(26.2 \%)\end{array}$ & $\begin{array}{l}39(13.1 \%) \\
10(9.7 \%)\end{array}$ & $\begin{array}{l}93(32.3 \%) \\
38(36.9 \%)\end{array}$ & $\begin{array}{l}40(13.5 \%) \\
28(27.2 \%)\end{array}$ & $\begin{array}{l}14.95 \\
\mathbf{0 . 0 0 2}\end{array}$ \\
\hline $\begin{array}{l}\text { Have you ever experienced } \\
\text { early or late periods before } \\
\text { your date in stressful } \\
\text { condition? } \\
\text { No } \\
\text { Yes }\end{array}$ & $\begin{array}{l}140(100 \%) \\
260(100 \%)\end{array}$ & $\begin{array}{l}67(47.9 \%) \\
85(32.7 \%)\end{array}$ & $\begin{array}{l}20(14.3 \%) \\
29(11.2 \%)\end{array}$ & $\begin{array}{l}35(25 \%) \\
96(36.9 \%)\end{array}$ & $\begin{array}{l}18(12.9 \%) \\
50(19.2 \%)\end{array}$ & $\begin{array}{l}12.36 \\
\mathbf{0 . 0 0 6}\end{array}$ \\
\hline $\begin{array}{l}\text { Menstrual Disorder } \\
\text { Normal } \\
\text { Oligomenorrhea } \\
\text { Polymenorrhagia } \\
\text { No fixed Pattern } \\
\end{array}$ & $\begin{array}{l}251(100 \%) \\
45(100 \%) \\
61(100 \%) \\
43(100 \%)\end{array}$ & $\begin{array}{l}106(42.2 \%) \\
10(22.2 \%) \\
18(29.5 \%) \\
18(41.9 \%)\end{array}$ & $\begin{array}{l}29(11.6 \%) \\
05(11.1 \%) \\
08(13.1 \%) \\
07(16.3 \%)\end{array}$ & $\begin{array}{l}80(31.9 \%) \\
16(35.6 \%) \\
21(34.4 \%) \\
14(32.6 \%)\end{array}$ & $\begin{array}{l}36(14.3 \%) \\
14(31.1 \%) \\
14(23 \%) \\
04(9.3 \%)\end{array}$ & $\begin{array}{l}15.51 \\
0.07\end{array}$ \\
\hline $\begin{array}{l}\text { Number of pads used } \\
1 \\
2-5 \\
>5\end{array}$ & $\begin{array}{l}67(100 \%) \\
273(100 \%) \\
60(100 \%) \\
\end{array}$ & $\begin{array}{l}23(34.3 \%) \\
115(42.1 \%) \\
14(23.3 \%) \\
\end{array}$ & $\begin{array}{l}8(11.9 \%) \\
36(13.2 \%) \\
05(8.3 \%) \\
\end{array}$ & $\begin{array}{l}25(37.3 \%) \\
81(29.7 \%) \\
25(41.7 \%) \\
\end{array}$ & $\begin{array}{l}11(16.4 \%) \\
41(15 \%) \\
16(26.7 \%)\end{array}$ & $\begin{array}{l}12.42 \\
0.05\end{array}$ \\
\hline $\begin{array}{l}\text { Days of menstrual bleeding } \\
\text { usually during each } \\
\text { period? } \\
\leq 2 \\
3-7 \\
>7 \\
\end{array}$ & $\begin{array}{l}12(100 \%) \\
358(100 \%) \\
30(100 \%)\end{array}$ & $\begin{array}{l}3(25 \%) \\
144(40.2 \%) \\
05(16.7 \%)\end{array}$ & $\begin{array}{l}0(0 \%) \\
43(12 \%) \\
06(20 \%)\end{array}$ & $\begin{array}{l}8(67 \%) \\
111(31 \%) \\
12(40 \%)\end{array}$ & $\begin{array}{l}1(8.3 \%) \\
60(16.8 \%) \\
7(23.3 \%)\end{array}$ & $\begin{array}{l}13.82 \\
0.03\end{array}$ \\
\hline $\begin{array}{l}\text { Dysmenorrhea } \\
\text { No dysmenorrhea } \\
\text { Mild dysmenorrhea } \\
\text { Moderate dysmenorrhea } \\
\text { Heavy dysmenorrhea } \\
\end{array}$ & $\begin{array}{l}35(100 \%) \\
117(100 \%) \\
161(100 \%) \\
87(100 \%) \\
\end{array}$ & $\begin{array}{l}17(48.6 \%) \\
51(43.6 \%) \\
61(37.9 \%) \\
23(26.4 \%) \\
\end{array}$ & $\begin{array}{l}5(14.3 \%) \\
14(12 \%) \\
18(11.2 \%) \\
12(13.8 \%) \\
\end{array}$ & $\begin{array}{l}8(22.4 \%) \\
39(33.3 \%) \\
51(31.7 \%) \\
33(37.9 \%) \\
\end{array}$ & $\begin{array}{l}5(11.4 \%) \\
13(11.1 \%) \\
31(19.3 \%) \\
19(21.8 \%) \\
\end{array}$ & $\begin{array}{l}11.84 \\
0.24\end{array}$ \\
\hline $\begin{array}{l}\text { Secondary Amenorrhea } \\
\text { No } \\
\text { Yes }\end{array}$ & $\begin{array}{l}334(100 \%) \\
66(100 \%)\end{array}$ & $\begin{array}{l}132(29.5 \%) \\
20(30.3 \%)\end{array}$ & $\begin{array}{l}43(12.9 \%) \\
06(9.1 \%)\end{array}$ & $\begin{array}{l}108(32.3 \%) \\
23(34.8 \%)\end{array}$ & $\begin{array}{l}51(15.3 \%) \\
17(25.8 \%)\end{array}$ & $\begin{array}{l}5.54 \\
0.13\end{array}$ \\
\hline $\begin{array}{l}\text { Did menstrual problems } \\
\text { affect your academic }\end{array}$ & & & & & & 14.37 \\
\hline
\end{tabular}


Biosight 2021; 02(02): 40-49

\begin{tabular}{|l|l|l|l|l|l|l|}
\hline performance? & $144(100 \%)$ & $72(50 \%)$ & $13(9 \%)$ & $40(27.8 \%)$ & $19(13.2 \%)$ & $\mathbf{0 . 0 0 3}$ \\
No & $256(100 \%)$ & $80(31.3 \%)$ & $36(14.1 \%)$ & $91(35.5 \%)$ & $49(19.1 \%)$ & \\
\hline
\end{tabular}

Association of categories of Stress with Menstrual Abnormalities.

Among study participants who experienced early or late periods before date in stressful condition, there was an increased prevalence of moderate stress $\left[\chi^{2}=14.12 \mathrm{p}\right.$ value $\left.<0.001\right]$. In moderately stressed females there was increased prevalence of polymenorrhagia $\left[\chi^{2}=22.3 p<0.001\right]$, increased duration of menstrual bleeding $\left[\chi^{2}=24.23 \mathrm{p}<0.0001\right]$, severe dysmenorrhea $\left[\chi^{2}=21.28 \mathrm{p}<0.01\right]$ and secondary amenorrhea $\left[\chi^{2}=4.28 \mathrm{p}<0.01\right]$. The academic performance of moderately stressed females was also disturbed $\left[\chi^{2}=12.66 \mathrm{p}\right.$ value $\left.<0.002\right]$ (Table 4).

Table 4. Association of categories of Stress with Menstrual Abnormalities.

\begin{tabular}{|c|c|c|c|c|c|}
\hline Factors & Frequency & $\begin{array}{l}\text { Normal } \\
\mathrm{n}=283 \\
(70.8 \%)\end{array}$ & $\begin{array}{l}\text { Mild } \\
n=90 \\
(22.5 \%)\end{array}$ & $\begin{array}{l}\text { Moderate } \\
\mathrm{n}=27 \\
(6.8 \%) \\
\end{array}$ & $\begin{array}{l}\chi^{2} \text { and } \\
\mathrm{p} \text { value }\end{array}$ \\
\hline $\begin{array}{l}\text { Did COVID-19 pandemic affect } \\
\text { your mental health? } \\
\text { No } \\
\text { Yes }\end{array}$ & $\begin{array}{l}161(100 \%) \\
239(100 \%)\end{array}$ & $\begin{array}{l}119(73.9 \%) \\
163(68.2 \%)\end{array}$ & $\begin{array}{l}32(19.9 \%) \\
59(24.7 \%)\end{array}$ & $\begin{array}{l}10(6.2 \%) \\
17(7.1 \%)\end{array}$ & $\begin{array}{l}1.32 \\
0.51\end{array}$ \\
\hline $\begin{array}{l}\text { Did COVID19 pandemic affect } \\
\text { your menstrual cycle? } \\
\text { No } \\
\text { Yes }\end{array}$ & $\begin{array}{l}297(100 \%) \\
103(100 \%)\end{array}$ & $\begin{array}{l}215(72.4 \%) \\
67(65 \%)\end{array}$ & $\begin{array}{l}69(23.2 \%) \\
22(21.4 \%)\end{array}$ & $\begin{array}{l}13(4.4 \%) \\
14(13.6 \%)\end{array}$ & $\begin{array}{l}10.33 \\
0.06\end{array}$ \\
\hline $\begin{array}{l}\text { Have you ever experienced early } \\
\text { or late periods before your date } \\
\text { in stressful condition? } \\
\text { No } \\
\text { Yes }\end{array}$ & $\begin{array}{l}140(100 \%) \\
260(100 \%)\end{array}$ & $\begin{array}{l}113(80.7 \%) \\
169(65 \%)\end{array}$ & $\begin{array}{l}25(17.9 \%) \\
66(24 \%)\end{array}$ & $\begin{array}{l}02(1.4 \%) \\
25(9.6 \%)\end{array}$ & $\begin{array}{l}14.12 \\
\mathbf{0 . 0 0 1}\end{array}$ \\
\hline $\begin{array}{l}\text { Menstrual Disorder } \\
\text { Normal } \\
\text { Oligomenorrhea } \\
\text { Polymenorrhagia } \\
\text { No fixed Pattern }\end{array}$ & $\begin{array}{l}251(100 \%) \\
45(100 \%) \\
61(100 \%) \\
43(100 \%)\end{array}$ & $\begin{array}{l}187(74.5 \%) \\
25(55.6 \%) \\
34(55.7 \%) \\
36(83.7 \%)\end{array}$ & $\begin{array}{l}49(19.5 \%) \\
13(28.9 \%) \\
23(73.7 \%) \\
6(14 \%)\end{array}$ & $\begin{array}{l}15(6 \%) \\
7(15.6 \%) \\
4(6.6 \%) \\
1(2.3 \%)\end{array}$ & $\begin{array}{l}22.30 \\
\mathbf{0 . 0 0 1}\end{array}$ \\
\hline $\begin{array}{l}\text { Number of pads used } \\
1 \\
2-5 \\
>5\end{array}$ & $\begin{array}{l}67(100 \%) \\
273(100 \%) \\
60(100 \%)\end{array}$ & $\begin{array}{l}43(64.2 \%) \\
199(72.9 \%) \\
40(66.7 \%)\end{array}$ & $\begin{array}{l}18(26.9 \%) \\
60(22 \%) \\
13(21.7 \%)\end{array}$ & $\begin{array}{l}6(9 \%) \\
14(5 \%) \\
7(11.7 \%)\end{array}$ & $\begin{array}{l}5.17 \\
0.27\end{array}$ \\
\hline $\begin{array}{l}\text { Days of menstrual bleeding } \\
\text { usually during each period? } \\
\leq 2 \\
3-7 \\
>7\end{array}$ & $\begin{array}{l}12(100 \%) \\
358(100 \%) \\
30(100 \%)\end{array}$ & $\begin{array}{l}4(33.3 \%) \\
262(73.21 \%) \\
16(53.3 \%)\end{array}$ & $\begin{array}{l}8(66.7 \%) \\
71(19.8 \%) \\
12(40 \%)\end{array}$ & $\begin{array}{l}0(0 \%) \\
25(7 \%) \\
2(6.7 \%)\end{array}$ & $\begin{array}{l}24.23 \\
\mathbf{0 . 0 0 0 1}\end{array}$ \\
\hline $\begin{array}{l}\text { Dysmenorrhea } \\
\text { No dysmenorrhea } \\
\text { Mild dysmenorrhea } \\
\text { Moderate dysmenorrhea } \\
\text { Heavy dysmenorrhea } \\
\end{array}$ & $\begin{array}{l}35(100 \%) \\
117(100 \%) \\
161(100 \%) \\
87(100 \%) \\
\end{array}$ & $\begin{array}{l}27(77 \%) \\
95(81.2 \%) \\
104(64.6 \%) \\
56(64.4 \%) \\
\end{array}$ & $\begin{array}{l}6(17.1 \%) \\
17(14.5 \%) \\
46(28.6 \%) \\
22(25.3 \%) \\
\end{array}$ & $\begin{array}{l}2(5.7 \%) \\
5(4.3 \%) \\
11(6.8 \%) \\
9(10.3 \%) \\
\end{array}$ & $\begin{array}{l}13.89 \\
\mathbf{0 . 0 3 1}\end{array}$ \\
\hline $\begin{array}{l}\text { Secondary Amenorrhea } \\
\text { No }\end{array}$ & $334(100 \%)$ & $242(72.5 \%)$ & $70(21 \%)$ & $22(6.6 \%)$ & 4.28 \\
\hline
\end{tabular}


Biosight 2021; 02(02): 40-49

\begin{tabular}{|l|l|l|l|l|l|}
\hline Yes & $66(100 \%)$ & $40(60.6 \%)$ & $21(31.8 \%)$ & $05(7.6 \%)$ & $\mathbf{0 . 0 1}$ \\
\hline $\begin{array}{l}\text { Did menstrual problems affect } \\
\text { your academic performance? } \\
\begin{array}{l}\text { No } \\
\text { Yes }\end{array}\end{array}$ & $144(100 \%)$ & $113(78.5 \%)$ & $29(20.1 \%)$ & $2(1.4 \%)$ & 12.66 \\
\hline
\end{tabular}

\section{DISCUSSION:}

It is well established that mental health problems affect menstrual health. This is the first ever study in the context of COVID-19 pandemic to assess menstrual abnormalities. COVID-19 has impacted every walk of life. The government-imposed restrictions to contain the COVID-19 are essential steps to control the spread of COVID-19. These restrictions include travel,social distancing, isolation, closure of public spaces. Yet, many people had to go outside to seek their livelihood and education. COVID-19 has an impact on the education of students. During COVID19 pandemic governments allowed use of hybrid mode of learning at universities which included 15 days physical classes and 15-day online classes. Seeking education during COVID-19 altered the conventional lifestyle of students. Many previously published studies have reported about impact of the COVID-19on mental health of students. COVID-19 has been associated with increased prevalence of depression, anxiety, stress, sleep abnormalities among female students. This study examined the psychological impact of COVID-19 on university students. Our results showed that perceived mental health problems were associated with increased menstrual health problems previous reports have shown a positive association of COVID-19 Menstrual Abnormalities. Previously conducted study have reported $25 \%$ of females had an increase in cycle length, $20 \%$ of study participants had decreased cycle length, $36 \%$ had changes in bleeding time and over 50\% experienced increase psychosocial menstrual symptoms such as mood changes lack of motivation and reduce concentration, $17 \%$ said they were worried about their menstrual cycle changes. Our results indicated that $7.5 \%$ females have increased in cycle length $>7$ days, $25 \%$ of females reported that COVID-19 has disturbed their menstrual health. Our study has found a positive association between COVID-19 impacted mental health and increase in menstrual abnormalities. A previously published pre-pandemic study showed that the prevalence of depression (mild to severe) was $19.5 \%$ and prevalence of anxiety (mild to extremely severe) was $43 \%[25]$.We found that the prevalence of depression (mild to severe) was $46.6 \%$ and anxiety (mild to extremely severe) was 62.1. Comparison of these pre and post pandemic studies showed that depression (mild to severe) has increased by $27 \%$ and anxiety (mild to extremely severe) has increased by $19 \%$. A study which was conducted in the same area before the pandemic showed that the prevalence of oligomenorrhea, polymenorrhagia, and no fixed pattern was $13 \%, 7 \%$, and $4 \%$ respectively[18]. Our data shows the prevalence of oligomenorrhea, polymenorrhagia, and no fixed pattern $11.25 \%, 15.25 \%$ and $10.75 \%$ respectively. This comparison showed that oligomenorrhea has been increased by $1.75 \%$, polymenorrhagia has been increased by $8.5 \%$ and no fixed pattern has been increased by $6.75 \%$, which suggest an association between COVID-19 impacted mental health and menstrual abnormalities. The studies conducted on menstrual abnormalities during pandemic are also consistent with our findings. For example, a study conducted on Turkish health professionals who were working during pandemic showed $12 \%$ of study participants had irregular cycles, $26.9 \%$ had change in duration of bleeding, $33.8 \%$ had changes in amount of bleeding, and $23.6 \%$ had increased dysmenorrhea[22]. A study conducted on American females (Aged 18-45 years) showed that 50\% of females had noticed changes in their menstrual cycle length and 34\% of females had noticed changes in duration of menses. In addition to this, females who had high stress scores had longer duration of menses and heavier bleeding compared to those who had moderate stress[26]. In our study we have noticed that $23 \%$ of females who were moderately stressed noticed changes in their menstrual cycle, $17 \%$ females 
who were moderately stressed noticed severe dysmenorrhea. Taken together, these results show the relationship of COVID-19 affected mental health has a negative effect on menstrual health.

\section{CONCLUSION:}

In conclusion, our study showed that COVID-19 affected mental health is associated with increased prevalence of Menstrual Abnormalities among university students which also affected their academic performance as well. If COVID-19 pandemic continues to affect the mental health of female students, this would have serious consequences for female students. The findings of this study may help in assessing the multi-dimensional effects of COVID-19. We suggest that the Government of Pakistan and its provincial governments should direct their health care systems to start a mental health support program for females in educational institutions. For such efforts the hiring of the graduates of psychology and physiology departments to start psychosocial counselling would be valuable. Life skill course should be included as a compulsory course in the first semester of each program taught at university level.

\section{Ethics approval and consent to participate}

Informed consent was obtained from all individual participants included in the study. Students agreed to participate in the study through the information page of questionnaire.

\section{Human And Animal Rights}

No animals were used in this study. The study on humans was conducted in accordance with the ethical rules of the Helsinki Declaration and Good Clinical Practice.

\section{Availability of Data And Materials}

None.

\section{Funding}

None.

\section{Conflict of Interest}

The authors declare no conflict of interest, financial or otherwise.

\section{Acknowledgements}

None.

\section{References:}

1. Organization WH. WHO Coronavirus (COVID-19) Dashboard: World Health Organization 2021 [cited 2021 05/06]. Available from: https://covid19.who.int/.

2. Lee JJTLC, Health A. Mental health effects of school closures during COVID-19. 2020;4(6):421.

3. Chaturvedi K, Vishwakarma DK, Singh NJC, review ys. COVID-19 and its impact on education, social life and mental health of students: A survey. 2021;121:105866.

4. Aqeel M, Abbas J, Shuja KH, Rehna T, Ziapour A, Yousaf I, et al. The influence of illness perception, anxiety and depression disorders on students mental health during COVID-19 outbreak in Pakistan: a web-based cross-sectional survey. 2021.

5. Tee ML, Tee CA, Anlacan JP, Aligam KJG, Reyes PWC, Kuruchittham V, et al. Psychological impact of COVID-19 pandemic in the Philippines. J Affect Disord. 2020;277:379-91. 10.1016/j.jad.2020.08.043

6. Fawaz M, Samaha A. E-learning: Depression, anxiety, and stress symptomatology among Lebanese university students during COVID-19 quarantine. Nurs Forum. 2021;56(1):52-7.

10.1111/nuf.12521 


\section{Biosight 2021; 02(02): 40-49}

7. Huang Y, Zhao NJPr. Generalized anxiety disorder, depressive symptoms and sleep quality during COVID-19 outbreak in China: a web-based cross-sectional survey. 2020;288:112954.

8. Aolymat IJTAJoTM, Hygiene. A cross-sectional study of the impact of COVID-19 on domestic violence, menstruation, genital tract health, and contraception use among women in Jordan. 2021;104(2):519.

9. Stanton AL, Lobel M, Sears S, DeLuca RSJJoC, Psychology C. Psychosocial aspects of selected issues in women's reproductive health: Current status and future directions. 2002;70(3):751.

10. Davydov DM, Shapiro D, Goldstein IB, Chicz-DeMet AJJoPR. Moods in everyday situations: effects of menstrual cycle, work, and stress hormones. 2005;58(4):343-9.

11. Laessle RG, Tuschi RJ, Schweiger U, Pirke KMJP. Mood changes and physical complaints during the normal menstrual cycle in healthy young women. 1990;15(2):131-8.

12. Yonkers KA, O'Brien PM, Eriksson E. Premenstrual syndrome. Lancet (London, England). 2008;371(9619):1200-10. 10.1016/s0140-6736(08)60527-9

13. Pearlstein T, Steiner M. Premenstrual dysphoric disorder: burden of illness and treatment update. Journal of psychiatry \& neuroscience : JPN. 2008;33(4):291-301.

14. Nillni YI, Wesselink AK, Hatch EE, Mikkelsen EM, Gradus JL, Rothman KJ, et al. Mental health, psychotropic medication use, and menstrual cycle characteristics. 2018;10:1073.

15. Varma P, Junge M, Meaklim H, Jackson MLJPiN-P, Psychiatry B. Younger people are more vulnerable to stress, anxiety and depression during COVID-19 pandemic: A global cross-sectional survey. 2021;109:110236.

16. Debowska A, Horeczy B, Boduszek D, Dolinski DJPM. A repeated cross-sectional survey assessing university students' stress, depression, anxiety, and suicidality in the early stages of the COVID19 pandemic in Poland. 2020:1-4.

17. Patsali ME, Mousa D-PV, Papadopoulou EV, Papadopoulou KK, Kaparounaki CK, Diakogiannis I, et al. University students' changes in mental health status and determinants of behavior during the COVID-19 lockdown in Greece. 2020;292:113298.

18. Dars S, Sayed K, Yousufzai Z. Relationship of menstrual irregularities to BMI and nutritional status in adolescent girls. Pak J Med Sci. 2014;30(1):141-4. 10.12669/pjms.301.3949

19. Ali MTS. Menstrual Practices, Misconceptions and Management Amongst Adolescents In An Urban Population, Karachi, Pakistan: University of Peshawar, Pakistan; 2006.

20. Yuksel B, Ozgor FJIJoG, Obstetrics. Effect of the COVID-19 pandemic on female sexual behavior. 2020;150(1):98-102.

21. Bruinvels G, Goldsmith E, Blagrove R, Simpkin A, Lewis N, Morton K, et al. Prevalence and frequency of menstrual cycle symptoms are associated with availability to train and compete: a study of 6812 exercising women recruited using the Strava exercise app. 2021;55(8):438-43.

22. ARINKAN SA, VURAL FJJoCO, Gynecology. Menstrual Cycle Characteristics of Healthcare Professionals Working at COVID-19 Pandemic Clinics. 2021;31(2):60-4.

23. Dean AG SK, Soe MM. . OpenEpi:Open Source Epidemiologic Statistics for Public

Health. USA 2013 [cited 2020 10/17]. Available from: www.OpenEpi.com.

24. Aslam N. Urdu translation of the DASS21 2021 [cited 2021 10/07]. Available from:

http://www2.psy.unsw.edu.au/dass/Urdu/Aslam.htm.

25. Rab F, Mamdou R, Nasir SJE-EMHJ, 14 , 126-133,. Rates of depression and anxiety among female medical students in Pakistan. 2008.

26. Ozimek N, Velez K, Anvari H, Butler L, Goldman KN, Woitowich NCJJoWsH. Impact of Stress on Menstrual Cyclicity During the Covid-19 Pandemic: A Survey Study. 2021. 